\title{
A model of the dwarf nova WX Cet
}

\author{
P. Rogoziecki ${ }^{1}$ and A. Schwarzenberg-Czerny ${ }^{2,1}$ \\ 1 Adam Mickiewicz University Observatory, ul. Sloneczna 36, 60-286 Poznań, Poland \\ 2 Copernicus Astronomical Center, ul. Bartycka 18, 00-716 Warsaw, Poland \\ e-mail: alex@camk.edu.pl
}

Received 15 April 2002 / Accepted 22 October 2002

\begin{abstract}
We analyse time-resolved spectroscopy of WX Cet, a dwarf nova of the WZ Sge class, by means of a dynamical model based on the measured $K_{1}$ and using the superhump period excess $\epsilon$ to constrain the mass ratio $q$ of this CB. From time resolved spectroscopy of Thorstensen et al. we determine orbital amplitude $K_{1}$ and separation $2 V_{\mathrm{d}}$ of the disk emission lines as well as the S-wave amplitude $A_{\mathrm{s}}$ and relative phase $\alpha$ of the hot spot. Our dynamical analysis, following Smak's inclination independent solution for WZ Sge, yields rather high $q$, hardly compatible with the superhump resonance condition within the accretion disc. Rejecting $K_{1}$ as affected by tidal and/or stream effects, we follow Patterson and determine $q=0.085$ from $q-\epsilon$ relation based on superhump dynamics. In this way we obtain likely masses of the components $M_{1}=0.55 \pm 0.15 M_{\odot}$ and $M_{2}=0.047 \pm 0.013 M_{\odot}$. From this result we argue that despite similarities to WZ Sge, WX Cet with its relatively massive secondary represents an earlier stage of mass transfer in a cataclysmic binary than WZ Sge.
\end{abstract}

Key words. stars: novae, cataclysmic variables - stars: dwarf novae - stars individual: WX Cet - stars individual: WZ Sge accretion, accretion disks

\section{Introduction}

Recently Patterson (1998, 2001, hereafter P98, P01) drew attention to the importance of the WZ Sge stars (or TOADs), an extreme subclass of SU UMa dwarf novae, in understanding of evolution of the cataclysmic binaries (CBs). On the face of evidence of a split in the short period tail of the superhump excess - orbital period relation, $\epsilon-P$, he postulated existence of two populations of short period dwarf novae, pre- and post-period bouncers. The nomenclature refers to the scenario in which CBs evolve through the minimum of the orbital period (Paczynski \& Sienkiewicz 1981). In SU UMa dwarf novae some outbursts, called superoutbursts, are particularly strong and reveal optical oscillations called superhumps. The periods of superhumps are longer than the orbital period $P$ by a fraction $\epsilon=\left(P_{\text {sh }}-P_{\text {orb }}\right) / P_{\text {orb }}$. The WZ Sge have particularly rare and powerful superoutbursts, and very short orbital periods. Textbook description of dwarf novae in general and SU UMa in particular may be found in Warner (1995). The WZ Sge stars as a group are discussed by Bailey (1979), Downes \& Margon (1981), O’Donoghue et al. (1991), Howell et al. (1995).

The superhumps are thought to occur by precession of an elliptically distorted accretion disk (Vogt 1979; Osaki 1985). The adopted theory of superhumps requires a 3:1 orbital resonance to occur within the accretion disk. Since tidal interaction

Send offprint requests to: $\mathrm{P}$. Rogoziecki, e-mail: progoz@moon. astro.amu.edu.pl cuts the disc radius at about 0.7 of the white dwarf Roche lobe, small $q$ is necessary for the lobe and disc to reach sufficient dimensions (Paczynski 1977; Whitehurst 1988; Osaki 1989; Lubow 1992). The superhump excess $\epsilon$ seems to depend primarily on $q$ (cf. P01 and references there). Thus verification of the two-branch hypothesis depends on reliable determination of $q$ and $M_{2}$ in WZ Sge stars.

WX Cet is a WZ Sge dwarf nova discovered during an outburst in 1963 (Strohmeier 1964). Many features of WX Cet make it very similar to WZ Sge itself. This similarity was noted by Bailey (1979), who first assigned it to WZ Sge-type dwarf novae. The superhump period in WX Cet was measured by O'Donoghue et al. (1991) and, finally, its spectroscopic orbital period was established securely by Thorstensen et al. (1996). Discovery of a photometric modulation in quiescence, a so-called orbital hump, enabled refinement of the orbital period and indicated that the inclination cannot be too low (Rogoziecki \& Schwarzenberg-Czerny 2001). Further observations and evidence supporting classification of WX Cet as a WZ Sge star were discussed by Downes \& Margon (1981), Downes (1990), Mennickent (1994) and Rogoziecki \& Schwarzenberg-Czerny (2001 and references therein).

In the present paper we discuss the results of spectroscopic observations of WX Cet in quiescence. Details of observations are given in Sect. 2. Using a method proposed by Smak (1993), we have performed spectral analysis of three emission lines. Thanks to this method we were able to determine some basic 

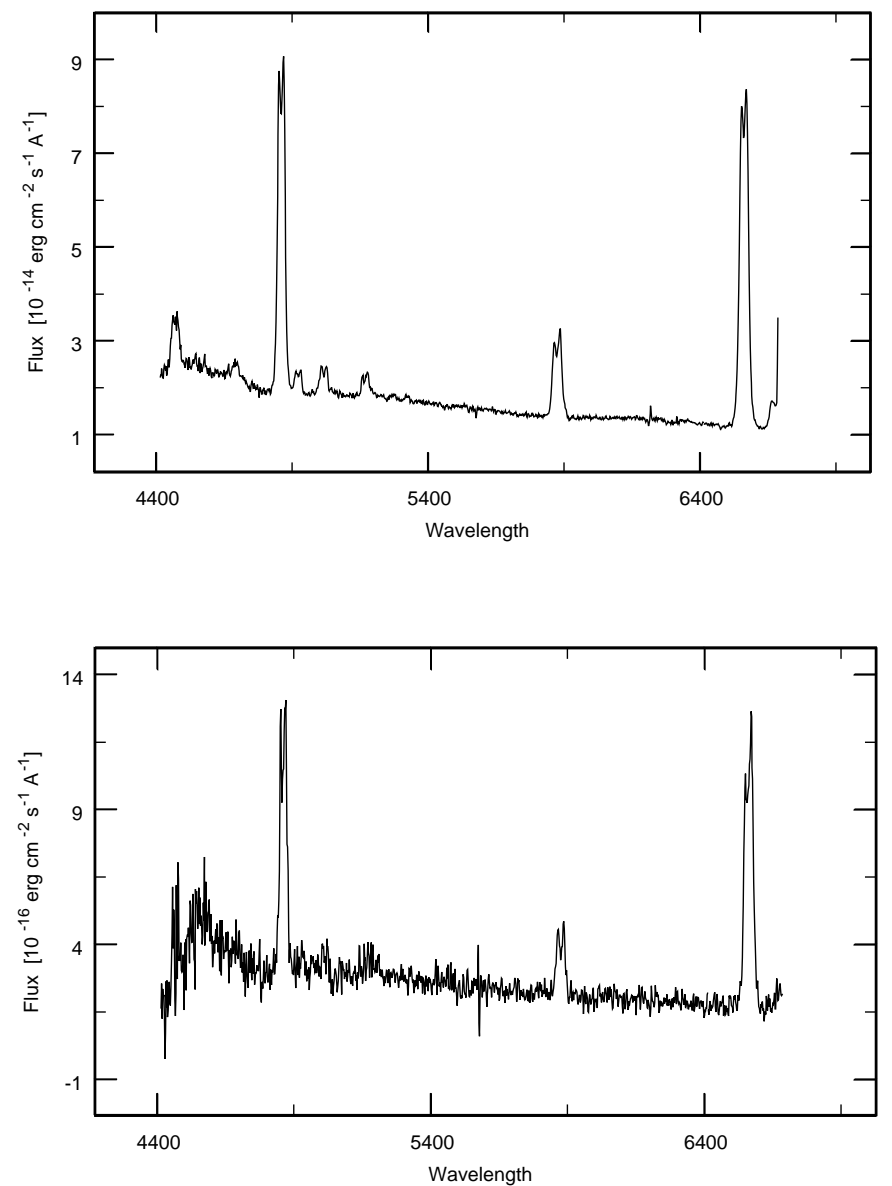

Fig. 1. Average and sample spectrum of WX Cet.

physical parameters of the WX Cet system as masses and sizes of the components, their separation and the radius of the disk. In Sect. 5 we compare WX Cet and WZ Sge.

\section{Emission line modeling}

\subsection{Data}

We analysed observations of WX Cet obtained by Thorstensen et al. (1996, hereafter TPST) using the MDM Observatory 2.4-m Hiltner reflector on Kitt Peak and Mark III spectrograph with a resolution of $5 \AA$ FWHM over the 4415 to $6687 \AA$ range. In total 67 spectra of WX Cet were collected on 3 nights in September 1993. Details of observations, reliable identification of the orbital period and $K_{1}$ measured from the line wings are given by TPST. For detailed analysis we chose three strongest lines in the observed range: $\mathrm{H} \alpha(6563 \AA), \mathrm{H} \beta(4861 \AA)$ and He I (5876 $\AA$ ) (Fig. 1). Plots of the line details reveal marked variation of the profiles over the orbital cycle (Fig. 2).

In order to emphasize the nature of the line profile variations we binned spectra in 13 phase bins, averaged them within bins and plotted them side-by-side in a density map fashion. In this way for three lines we obtain a digital analogue of a single trailed photographic spectrum. A sample spectrum for the $\mathrm{H} \beta$ line is shown in Fig. 3. All trailed spectra reveal two well-separated components from the accretion disk with a superposed S-wave component. For the dynamical solution we

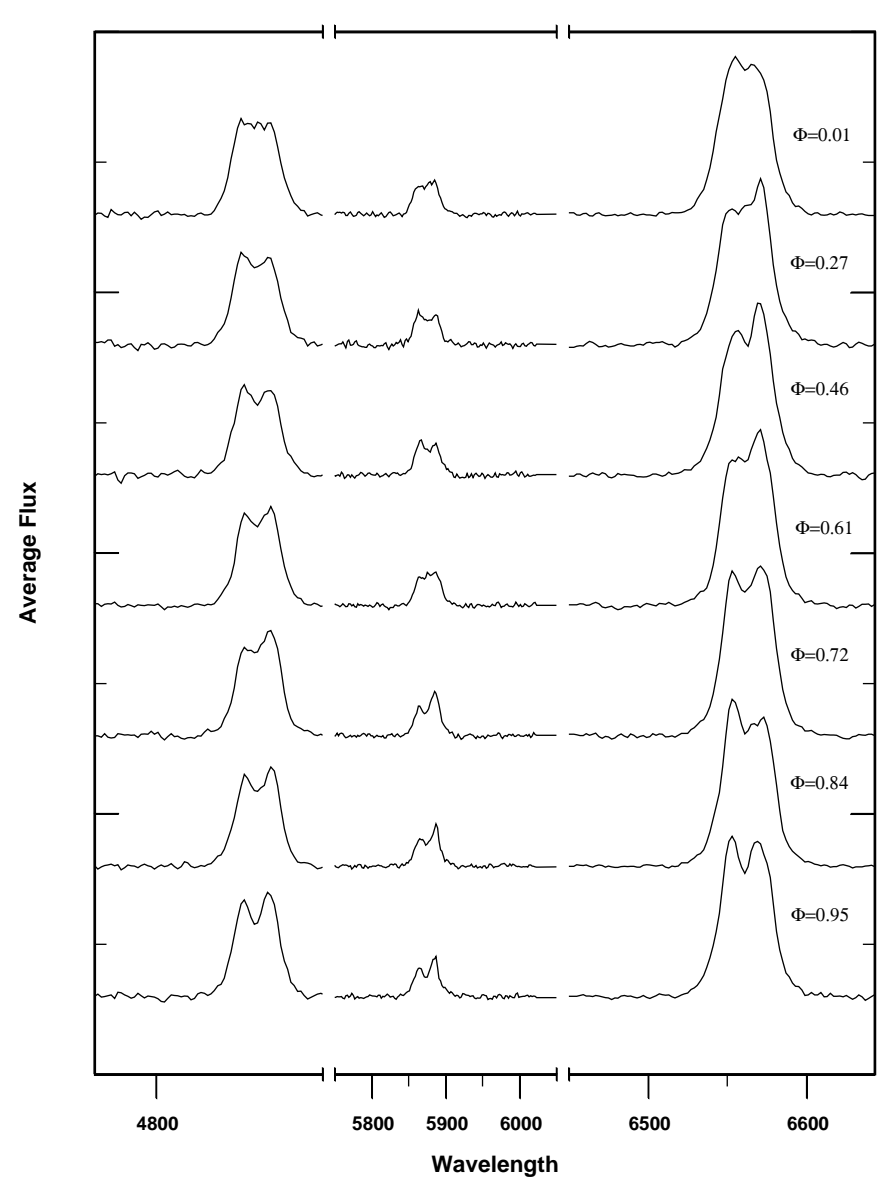

Fig. 2. Phase binned spectra for lines (from left) $\mathrm{H} \beta$, He I and $\mathrm{H} \alpha$. Each bin is labeled with the average orbital phase.

need the orbital amplitude $K_{1}$ and separation $2 V_{\mathrm{d}}$ of the disk emission lines as well as the $\mathrm{S}$-wave amplitude $A_{\mathrm{s}}$ and the phase $\alpha$ of the hot spot. For this purpose we fitted the density map with a three-component line model defined in Sect. 2.2, using nonlinear least-squares methods.

\subsection{The three component line model}

We employ the following model for the red, blue and S-wave components, respectively:

$F_{\mathrm{d}}(1)=\frac{1}{\sqrt{2 \pi} \sigma_{\mathrm{d}}} \exp \left\{\frac{-\left[\lambda-\gamma_{\mathrm{d}}-V_{\mathrm{d}}-K_{1} \sin 2 \pi\left(\varphi-\varphi_{\mathrm{d}}\right)\right]^{2}}{2 \sigma_{\mathrm{d}}^{2}}\right\}$

$F_{\mathrm{d}}(2)=\frac{1}{\sqrt{2 \pi} \sigma_{\mathrm{d}}} \exp \left\{\frac{-\left[\lambda-\gamma_{\mathrm{d}}+V_{\mathrm{d}}-K_{1} \sin 2 \pi\left(\varphi-\varphi_{\mathrm{d}}\right)\right]^{2}}{2 \sigma_{\mathrm{d}}^{2}}\right\}$

$F_{\mathrm{s}}=\frac{1}{\sqrt{2 \pi} \sigma_{\mathrm{s}}} \exp \left\{\frac{-\left[\lambda-\gamma_{\mathrm{s}}+A_{\mathrm{s}} \sin 2 \pi\left(\varphi-\varphi_{\mathrm{s}}\right)\right]^{2}}{2 \sigma_{\mathrm{s}}^{2}}\right\}$

The $F_{\mathrm{d}}(1)$ and $F_{\mathrm{d}}(2)$ arise from the approaching and receding parts of the disk, and $F_{\mathrm{s}}$ is an $\mathrm{S}$-wave component due to the hot spot. These profiles are combined in our final model $F$ using

$F=(1-\epsilon)\left[F_{\mathrm{d}}(1)+F_{\mathrm{d}}(2)\right]+\epsilon F_{\mathrm{s}}$. 


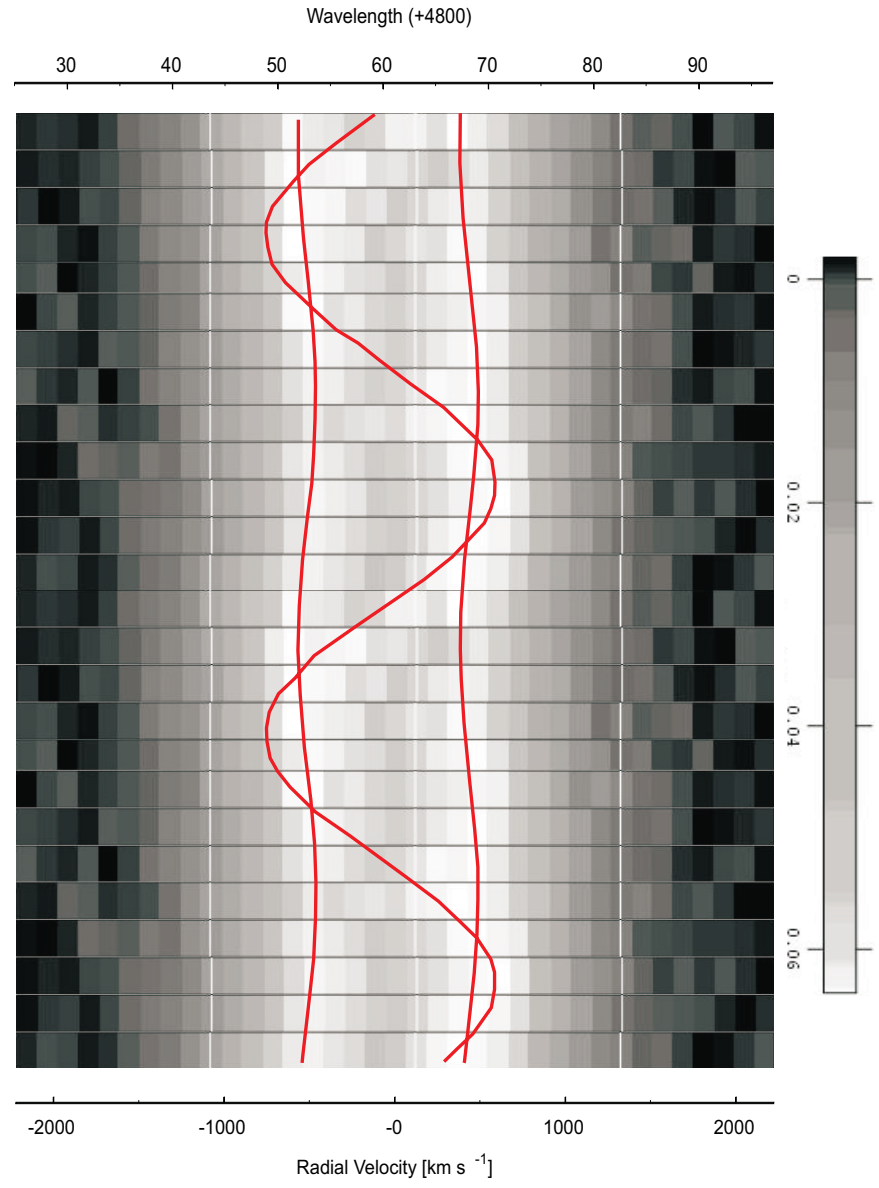

Fig. 3. Trailed spectra for line $\mathrm{H} \beta$. Note that data are repeated over two orbital cycles.

With $\gamma_{\mathrm{d}, \mathrm{s}}, \varphi_{\mathrm{d}, \mathrm{s}}, \sigma_{\mathrm{s}, \mathrm{d}}$ we denote the gamma velocities, absolute phases and line width, for the disk and S-wave components, respectively. Eventually our fits yielded parameters designated $K_{1}, V_{\mathrm{d}}, \varphi_{\mathrm{d}}$ for the disk and $A_{\mathrm{s}}, \varphi_{\mathrm{s}}$ for the $\mathrm{S}$-wave listed in Table 1, for each line separately. The dynamical solution depends on the difference $\alpha=\varphi_{\mathrm{s}}-\varphi_{\mathrm{d}}$, called hereafter for brevity the phase. At this point we caution that the measured parameters may be affected by systematic errors to be discussed in Sect. 2.3.

We conclude this section with discussion of instrumental and reduction errors. We performed experiments aimed at evaluation of robustness of our derived $K_{1}$. Separate sinusoids were obtained for the red and blue components with sinusoids, for the original trailed spectrum and the spectrum with the S-wave subtracted. All gave $K_{1}$ and $\varphi_{\mathrm{d}}$ mutually consistent between components, and consistent with the quoted values, too. In this way we convinced ourselves that the presence of a strong $\mathrm{S}$-wave does not interfere much with the measurements of $K_{1}$.

\subsection{Discussion of systematic errors}

The patterns seen in Fig. 3 and similar figures observed in other $\mathrm{CBs}$ is peculiar and difficult to explain without reference to kinematic effects of the orbital motion, rotation of the disk and flow of the stream. This said we must remember that the $K_{1}$ measurements failed two consistency checks, as noted by P01. In the eclipsing CBs, spectroscopic phases of $K_{1}$ were observed to depart from those consistent with eclipses by $15^{\circ}$ on average and quadruple that much at worst. In the best studied short period $\mathrm{CB}$, WZ Sge, observations with different instruments yielded over factor 2 discordance in $K_{1}: K_{1}<38, K_{1}=49$ and $K_{1}<20 \mathrm{~km} \mathrm{~s}^{-1}$ (Krzeminski \& Kraft 1964; Gilliland et al. 1986; and more recently Cheng et al. 1997). Note, however, that Smak (1993) considered the high value given by Gilliland et al. (1986) as realistic, and derived a self-consistent dynamical model with the mass of the white dwarf in WZ Sge as low as $M_{1}=0.45 M_{\odot}$.

Our discussion of these systematic errors may be summarised in the following points: (i) TPST measured wings of lines. The wings are produced in the inner disk where orbits are perfectly circular and therefore their measurements do not suffer from the tidal distortion. They may suffer from the stream component as argued by Smak (2001a). (ii) We measured $K_{1}$ from the peaks of lines, independently for the blue and red components. In our measurements we do account explicitly for the principal distortion of disc lines by fitting the S-wave originating from the hot spot. Any residual hot spot effect and the tidal effect should affect the measurements (i) and (ii) differently and at different phases. (iii) Our red and blue $K_{1}$ agree. This demonstrates that the measurements from two halves of disk are free of hot spot effects or the hot spot effects conspire to yield similar distortions in geometrically quite different locations. A symmetric tidal distortion is still possible. (iv) Despite use of different methods our and TPST $K_{1}$ results agree within errors, yielding $K_{1}=56 \pm 3$ and $K_{1}=57 \pm 2$, respectively.

None of the points (i)-(iv) prove lack of severe distortion of $K_{1}$ measurement. They indicate though that these systematic errors must conspire to a large degree to yield similar values from rather different effects. This point is worth of investigation in other stars/measurements as some real physics may hide behind this effect.

\section{Dynamic models}

For the consistency check of $K_{1}$ we attempt dynamical modeling of the observed velocities and phases. We use average $K_{1}$, $V_{\mathrm{d}}$ and $A_{\mathrm{s}}$ from Table 1 to fit models in Sects. 3.2 and 3.3 in a way which is independent of $i$.

\subsection{Smak's dynamic solution}

Smak's (1993) model assumes a Keplerian accretion disk revolving around the white dwarf orbiting the binary mass centre. The hot spot is assumed to lie at certain radius along the stream of gas flowing from the $L_{1}$ point towards the disk, and possibly skidding above the surface of the disk. In the latter case the hot spot could be located at a radius inside of the disk. Adopting the orbital period $P / 2 \pi=1$ and separation of components $a=1$ for units of time and length, Smak's (1993) Eqs. (1), (2), (6) and (5) may be written as:

$$
\frac{\tilde{K}_{1}}{\sin i}=\mu
$$


Table 1. Fitted model parameters.

\begin{tabular}{ccccccc}
\hline \hline Line & $\gamma_{\mathrm{d}}\left(\mathrm{km} \mathrm{s}^{-1}\right)$ & $K_{1}\left(\mathrm{~km} \mathrm{~s}^{-1}\right)$ & $V_{\mathrm{d}}\left(\mathrm{km} \mathrm{s}^{-1}\right)$ & $A_{\mathrm{s}}\left(\mathrm{km} \mathrm{s}^{-1}\right)$ & $\varphi_{\mathrm{d}}$ & $\varphi_{\mathrm{s}}$ \\
\hline $\mathrm{H} \alpha$ & $-35 \pm 1$ & $51 \pm 2$ & $441 \pm 1$ & $722 \pm 21$ & $0.370 \pm 0.007$ & $0.559 \pm 0.004$ \\
$\mathrm{H} \beta$ & $-17 \pm 2$ & $56 \pm 3$ & $497 \pm 2$ & $707 \pm 28$ & $0.414 \pm 0.011$ & $0.592 \pm 0.005$ \\
He I & $-39 \pm 4$ & $62 \pm 6$ & $535 \pm 3$ & $607 \pm 15$ & $0.365 \pm 0.016$ & $0.583 \pm 0.003$ \\
\hline average & $-30 \pm 2$ & $56 \pm 4$ & $491 \pm 2$ & $679 \pm 21$ & $0.383 \pm 0.011$ & $0.578 \pm 0.003$ \\
\hline
\end{tabular}

$$
\begin{aligned}
& \frac{\tilde{V}_{\mathrm{d}}}{\sin i}=\sqrt{\frac{1-\mu}{r_{\mathrm{d}}}} \\
& \frac{\tilde{A_{\mathrm{s}}}}{\sin i}=f\left(\mu, r_{\mathrm{s}}\right) \\
& \alpha=f\left(\mu, r_{\mathrm{s}}\right)
\end{aligned}
$$

where $\mu=M_{2} /\left(M_{1}+M_{2}\right)$ and $r_{\mathrm{d}}$ and $r_{\mathrm{s}}$ are outer radius of the disc and radius of location of the hot spot in units of $A$. With $\approx$ we denote the velocities expressed in our new units. Note that in order to simplify factors arising from unit conversion we substituted into Eq. (6) Kepler's 3rd law. Our definitions deviate here from Smak (1993) in that the $\sin i$ factors are included in $V_{\mathrm{d}}$ and $A_{\mathrm{s}}$. Functions $f(\cdot)$ are different for each equation and represent results of the stream trajectory numerical integration in the approximation of a free particle moving in the Roche potential.

\subsection{Hot spot on disk's rim}

Smak's (1993) solution for WZ Sge was obtained assuming $r_{\mathrm{d}}=r_{\mathrm{s}}$. Then by dividing Eqs. (5) and (7) by Eq. (6) we obtain

$$
\begin{aligned}
\frac{K_{1}}{V_{\mathrm{d}}} & =\sqrt{\frac{\mu^{2} r_{\mathrm{d}}}{1-\mu}} \\
\frac{A_{\mathrm{s}}}{V_{\mathrm{d}}} & =f\left(\mu, r_{\mathrm{d}}\right) .
\end{aligned}
$$

Values of functions on the right-hand sides are conveniently tabulated by Smak (1993, and extended table by private communication). They are plotted in Fig. 4 as a grid labeled with $\mu$ and $r_{\mathrm{d}}$. The left-hand sides are independent of units of velocities hence we skipped $\sim$ symbols. The left-hand sides of the new equations are known from observations hence for a given $K_{1} / V_{\mathrm{d}}$ and $A_{\mathrm{s}} / V_{\mathrm{d}}$, the whole system may be solved for $\mu$ and $r_{\mathrm{d}}$, e.g. graphically by using Fig. 4. In this figure, we marked with crosses points corresponding to Smak's (1993) solution for WZ Sge and our solution for WX Cet $\mu=0.34$ and $r_{\mathrm{d}}=0.08$. Our solution for WX Cet does not appear consistent with standard properties of accretion disks. Observed disk radii tend to be as large as 0.7 of the corresponding Roche lobe, i.e. $r_{\mathrm{d}} \sim 0.3$, in order to remove excess angular momentum by the tidal action (Paczynski 1977). Up to this point no use was made of Eq. (8). By substitution of solutions into Eq. (8) for WZ Sge, Smak obtained $\alpha$ consistent with observations while for WX Cet we obtain $\alpha$ inconsistent with the observed one. On these grounds we reject the model with $r_{\mathrm{d}}=r_{\mathrm{s}}$.

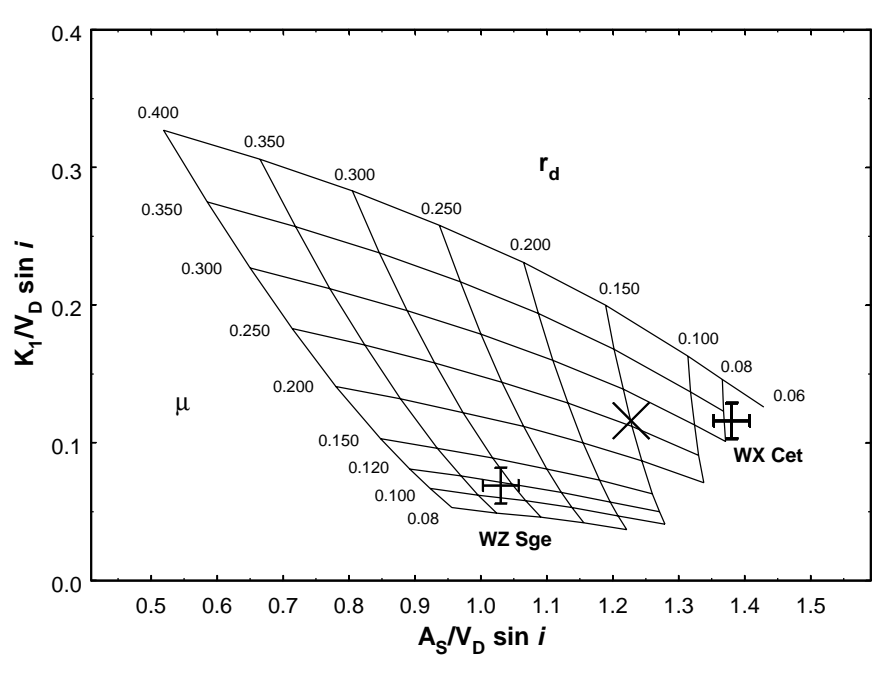

Fig. 4. The $K_{1} / V_{\mathrm{d}} \sin i$ vs. $A_{\mathrm{s}} / V_{\mathrm{d}} \sin i$ diagram, with a grid of theoretical lines parameterized with $r_{\mathrm{d}}$ and $\mu$. The "+" signs mark ordinary solutions for WZ Sge and WX Cet, the "X" sign marks WX Cet solution accounting for penetration of the disc by the stream.

\subsection{Hot spot inside disk}

In general the hot spot may be located inside of disc, for $r_{\mathrm{s}}<r_{\mathrm{d}}$. This may happen for example if the stream manages to split and slide above and under the faces of the disk. So we relax here the condition $r_{\mathrm{d}}=r_{\mathrm{s}}$. Now we are faced with three unknowns $\mu, r_{\mathrm{d}}$ and $r_{\mathrm{s}}$ and a system of three equations

$$
\begin{aligned}
& \frac{K_{1}}{V_{\mathrm{d}}}=\sqrt{\frac{\mu^{2} r_{\mathrm{d}}}{1-\mu}} \\
& \frac{A_{\mathrm{s}}}{V_{\mathrm{d}}}=f\left(\mu, r_{\mathrm{d}}, r_{\mathrm{s}}\right) \\
& \alpha=f\left(\mu, r_{\mathrm{s}}\right) .
\end{aligned}
$$

Note that Eqs. (9), (8) and (11), (13) are identical. By expressing the left-hand side of Eq. (12) as $\left(A_{\mathrm{s}} / V_{\mathrm{s}}\right)\left(V_{\mathrm{s}} / V_{\mathrm{d}}\right)=$ $\left(A_{\mathrm{s}} / V_{\mathrm{s}}\right) \sqrt{r_{\mathrm{d}} / r_{\mathrm{s}}}$, we note that it could be evaluated from tabulation of Eq. (10) scaled by the factor $\sqrt{r_{\mathrm{d}} / r_{\mathrm{s}}}$. Thus the solution of the general case $r_{\mathrm{s}} \neq r_{\mathrm{d}}$ may be obtained using functions tabulated for the special case $r_{\mathrm{s}}=r_{\mathrm{d}}$.

The system of Eqs. (11), (12) and (13) may be solved for $\mu$, $r_{\mathrm{d}}$ and $r_{\mathrm{s}}$. First we solve analytically Eq. (11) for $r_{\mathrm{d}}$ and substitute the solution $r_{\mathrm{d}}=f(\mu)$ into Eqs. (12) and (13). Next we adopt a graphical method of solution and use the right-hand side of the remaining system of Eqs. (12) and (13) to plot in Fig. $5 A_{\mathrm{s}} / V_{\mathrm{d}}$ against $\alpha$ for a grid of $\mu$ and $r_{\mathrm{s}}$. For the observed values of $A_{\mathrm{s}} / V_{\mathrm{d}}$ and $\alpha$ from Fig. 5 we read for WX Cet $\mu=0.26$ and $r_{\mathrm{s}}=0.13$. Then from Eq. (11) we obtain $r_{\mathrm{d}}=0.15$. 


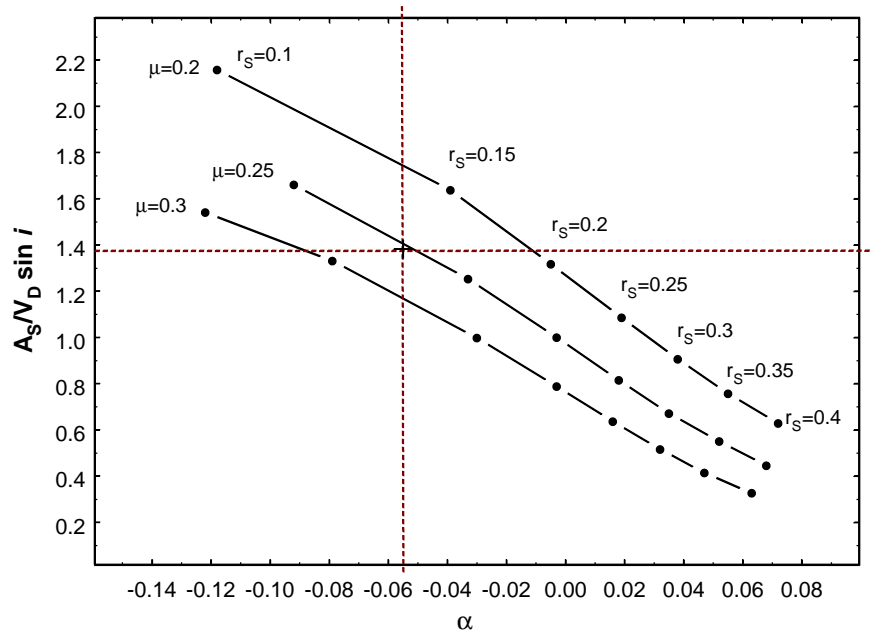

Fig. 5. The relative S-Wave amplitude $A_{\mathrm{s}} / V_{\mathrm{d}} \sin i$ vs. S-wave phase $\alpha$ diagram, with a set of theoretical lines parameterized with $\mu$ and $r_{\mathrm{s}}$. The observational values give two crossed dashed lines.

The corresponding values of $\mu$ and $r_{\mathrm{d}}$ are marked in Fig. 4 with a X mark. In any case $\mu$ derived for WX Cet from dynamical models is large compared to that for WZ Sge. We shall discuss the reliability of our dynamical model in Sect. 5.

Note that differences in physics of WX Cet and WZ Sge may be appreciated without reference to any dynamical models, by simple comparison of our the trailed spectra of WX Cet (Fig. 3) and those of WZ Sge (e.g. Skidmore et al. 2000, their Fig. 1). In WX Cet the amplitude of S-wave $A_{\mathrm{S}}$ is large compared to the half peak separation $V_{\mathrm{d}}$. Particularly noticeable in WX Cet are excursions of the S-wave beyond the red disk component. No such effect is seen in WZ Sge. In these figures the binary inclination affects only the units on the horizontal scale, and may not explain marked differences in shapes.

\section{Evidence from superhumps}

\subsection{General restrictions}

The simple existence of superhumps in WX Cet poses limits on the system parameters. The superhump theory reviewed in Sect. 1 demands $q<0.25$. In practice normal superhumps were observed in UU Aqr with $q=0.30$ and the long orbital period of $P_{\text {orb }}=0.16 \mathrm{~d}$ (Baptista et al. 1994; P01). This discrepancy may be due to the neglect by theoreticians of pressure effects. The large $q=0.35$ following from our dynamical solution appears to lie on the wrong side of the limit and is not consistent with the short orbital period. Additionally, combined with other restrictions this large $q$ would demand a white dwarf with very low mass, difficult to produce in the standard evolutionary scenario (Sect. 5). The permanent superhumps are also observed in TV Col with $P_{\text {orb }}$ as large as $0.23 \mathrm{~d}$, and correspondingly large $q$. However, the presence of superhumps there may be affected by interaction of the disc with the magnetosphere of the magnetic white dwarf (Augusteijn et al. 1994).

Our large $q$ follows mainly from the large observed $K_{1}$. There are reasons to doubt whether the measured $K_{1}$ and its corresponding large $q$ in the very short period binaries are real
(P01), especially since tidal and/or stream perturbation of the disk's rim by less then $10 \%$ of its rotational velocity would completly obscure any orbital effect.

\subsection{Mass ratio from superhump excess}

An alternate approach to finding system parameters is to employ the mass ratio-superhump period excess, $q-\epsilon$ relation as proposed by $\mathrm{P} 01$. The orbital period and superhump period, $P_{\text {orb }}$ and $P_{\text {sh }}$ of WX Cet are known from observations (TPST; Rogoziecki \& Schwarzenberg-Czerny 2001; O'Donoghue et al. 1991) and $\epsilon=P_{\mathrm{sh}} / P_{\mathrm{orb}}-1$. From Eq. (5) of P01 we obtain $q=0.085 \pm 0.009$, where the error accounts for the scatter of the $q-\epsilon$ relation (mostly resulting from wandering of $P_{\mathrm{sh}}$ ). Small as this $q$ is, it is twice as large that estimated by P01 for WZ Sge. Thus despite similarities, the mass ratio of WX Cet is factor 2 bigger than for WZ Sge, regardless of whether both are derived from $K_{1}$ or $\epsilon$. In this approach no direct estimate of the disc radius is available. We shall assume that the radius in quiescence is close to the superhump resonance radius, found empirically by P01 to be close to $r_{\mathrm{d}}=0.37 a$. The hope is that by assuming so we err at least consistently by the same factor for all superhump CBs.

\section{Discussion}

In this section we use $q$ and $r_{\mathrm{d}}$ derived in Sects. 3 and 4 in order to derive masses and inclinations and to discuss other properties of the binary.

\subsection{Masses and inclination}

Using additional information on the disk rotation velocity $V_{\mathrm{d}}$ we may derive masses. We return here to the ordinary units of time and length, so that Eq. (5) assumes the following form:

$\frac{V_{\mathrm{d}}}{\sin i}=\frac{2 \pi a}{P} \sqrt{\frac{1-\mu}{r_{\mathrm{d}}}}$.

Combining it with Kepler's third law and solving for $M_{1}$ and $a$ we obtain:

$$
\begin{aligned}
& M_{1}=\frac{V_{\mathrm{d}}^{3} P \sqrt{1+q}}{2 \pi G} \frac{r_{\mathrm{d}}^{3 / 2}}{\sin i^{3}} \\
& a=\left[G M_{1}(1+q)\right]^{1 / 3}\left(\frac{P}{2 \pi}\right)^{2 / 3} \\
& M_{2}=q M_{1} .
\end{aligned}
$$

Note that $M_{1}$ is a weak function of $q$ and hence depends weakly on whether superhump or dynamic solutions are used. It does depend on disc radius, however. Small disc radii prevent tidal braking and hence they are excluded by angular momentum conservation arguments. Paczynski (1977) argued that tidal braking increases sharply when Keplerian orbits start intersecting, and obtained an upper limit for disc radius of about 0.7 size of the Roche lobe. Emission lines originate from a ring of finite thickness, so they yield an average radius less than the radius of the disk rim. In that sense the disk radius $r_{\mathrm{d}}=0.31$ 
derived for WZ Sge by Smak (1993) from his dynamic solution is consistent with the accretion physics. Note that for the present case of small $q$, the location of the orbit intersection and stream trajectory depend weakly on $q$. Hence it is justified to assume that both WX Cet and WZ Sge contain disks of similar relative size. Any systematic error introduced by this assumption should cancel in a comparison of white dwarf masses. The solutions for masses of components as function of inclination $i$ for $r_{\mathrm{d}}=0.31$ are plotted in Fig. 6 as inclined lines. The two lines correspond to dynamical $\left(K_{1}\right)$ and superhump solutions for $q$. Inclinations $i$ are indicated by labeling. Acceptable solutions must obey several conditions. Lack of eclipses indicates $i<70^{\circ}$. Adopting the revised mass-radius relation one obtains the following mass-period relation (Clemens et al. 1998; P98):

$M_{2} \approx 0.048 P^{1.64}=0.083 M_{\odot}$,

where $P$ is the orbital period in hours. This is less than given by Eq. (2.89) of Warner (1995): $M_{2}=0.147 M_{\odot}$. The $M_{2}$ corresponding to the main sequence secondary constitutes the upper limit. An evolved (oversized) secondary would need to have less mass in order to fit its Roche lobe. Such an oversized secondary remains a viable possibility, particularly near the minimum period of $\sim 80 \mathrm{~min}$, where systems evolve faster than the secondary's thermal time scale (Paczynski \& Sienkiewicz 1981).

For $q$ derived from $K_{1}$ and a revised main sequence we obtain $M_{1} \sim 0.2 M_{\odot}$, possibly in conflict with the minimum evolutionary mass of the helium white dwarf able to evolve within age of the Galaxy, of about $0.15-0.25 M_{\odot}$ (Paczynski, Yungelson - private communication; Livio \& Iben 1993). This limit for $M_{1}$ is also indicated in Fig. 6. A massive secondary is unlikely as it demands small inclinations incompatible with the orbital modulation in quiescence being caused by an aspect effect. It is quite difficult to see what else could cause this modulation, hence the inclination must remain at least moderate, say $i \geq 40^{\circ}$. For this and other reasons discussed in P01 and in Sect. 4 we consider the low $q$ obtained from the superhump excess $\epsilon$ as more realistic.

Clearly, no aspect modulation occures for $i=0$ inclination. However, precise estimation of the limit $i$ consistent with the observed in WX Cet peak-to-peak modulation of $0.15 \mathrm{mag}$ is difficult. We resort to a crude scaling procedure. Namely, the luminosity of the disc scales with $\cos i$ (e.g. Paczynski \& Schwarzenberg-Czerny 1980). Motivated by symmetry arguments we asume that the modulated radiation of the hot spot $\Delta I$ comes from a plane surface perpendicular to the orbital plane, hence $\Delta I \sim \sin i$. If so, that the amplitude of modulation in magnitudes should roughly scale with $\tan i$. This crude approximation should hold at least for $i \ll 90^{\circ}$. By setting for U Gem $i=69^{\circ}$ and $\Delta I / I \approx 0.3 \mathrm{mag}$ (Smak 2001b and references there), for $\Delta I / I \approx 0.15 \mathrm{mag}$ in WX Cet we obtain $i>50^{\circ}$. Given crudness of the whole procedure we relax this condition to $i>40^{\circ}$.

Very small masses are excluded as no eclipses were detected, hence $i<70^{\circ}$. In fact, $i$ should be less than that limit as a very oversized, i.e. low mass secondary does not seem to be consistent with current evolutionary calculations. Hence we marked with the thick line the most likely masses, consistent

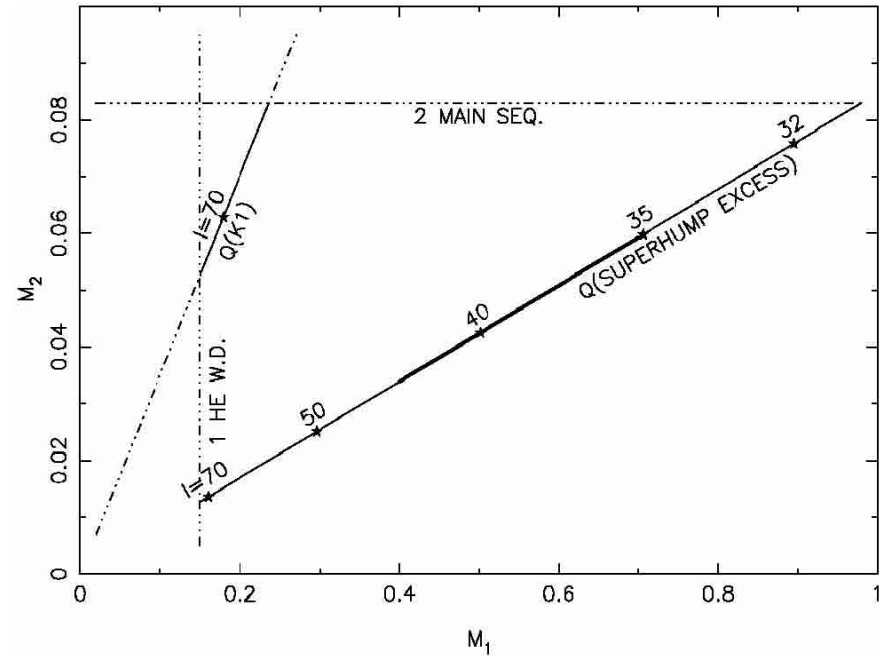

Fig. 6. The mass solutions for WX Cet. Inclined lines labeled with inclination $i$ correspond to dynamical and superhump excess solutions derived from $K_{1}$ radial velocity and superhump excess $\epsilon$, respectively. The thick line, corresponding to the mass ratio $q=0.085$ marks locus of the most probable masses of the components. Dotted lines indicate minimum mass of a post-common envelope helium white dwarf and theoretical maximum $q$ for superhumps.

with moderate inclinations $35^{\circ}<i<45^{\circ}$ and low $q$ derived from $\epsilon$. The corresponding masses are $M_{1}=0.55 \pm 0.15$ and $M_{2}=0.047 \pm 0.013 M_{\odot}$. Note that the white dwarfs in WX Cet and WZ Sge appear to have masses less than the average for CBs, $0.7 M_{\odot}$.

On the one hand, statistical analysis led P01 to doubt whether in extremely short period cataclysmic binaries the measured $K_{1}$ reflect actual kinematics. On the other hand, for WZ Sge Smak (1993) faced no difficulty in fitting $K_{1}$ with a self-consistent dynamic model. However, for WX Cet dynamical models based on $K_{1}$ appear to be less successful. This caused us to favour solutions bases on superhump $q-\epsilon$ correlation. However we note that rather fine tuning of systematic effects is required to explain consistency of the wing and peak $K_{1}$ measurements. This point merits investigation in other short period CBs. Clearly more measurements and more dynamical solutions of the type presented here are required to decide their reality on a star by star basis.

\subsection{WX Cet versus WZ Sge}

In Table 2 we list parameters derived for WZ Sge against those of WX Cet. For consistency with our approach we list as first values for WZ Sge derived by Smak (1993) using $K_{1}$. Recently during superoutburst Steeghs et al. (2001) obtained reliable $K_{2}$ and solid limits for small $q$ and large $M_{1}$. Following 2001 we refrain here from the temptation to rederive new parameters for WZ Sge till reliable $K_{1}$ are derived. The differences of the two sets indicate direction and magnitude of the involved systematic errors. Observation of $K_{2}$ for much fainter and rare outbursts outburst of WX Cet would be difficult.

Significant differences between WZ Sge and WX Cet may be perceived already by inspection of their trailed 
Table 2. Comparison of the parameters.

\begin{tabular}{lcr}
\hline \hline & WZ Sge & WX Cet \\
\hline$P_{\text {orb }}[\mathrm{min}]$ & 81.63 & 83.91 \\
$P_{\text {sh }}[\mathrm{min}]$ & 82.28 & 85.48 \\
$\epsilon$ & $\sim 0.80 \%$ & $\sim 1.87 \%$ \\
humps & 2 or 1 & 1 or 2 \\
$q$ & $\sim 0.13:^{a}(0.055 \pm 0.015)^{b}$ & $\sim 0.35^{c}$ \\
$q^{d}$ & $\sim 0.045$ & $\sim 0.085$ \\
$V_{\mathrm{d}}\left[\mathrm{km} \mathrm{s}^{-1}\right]$ & 710 & 497 \\
$r_{\mathrm{d}}[\mathrm{a}]$ & 0.31 & $0.31^{e}$ \\
eclipses & shallow & none $(?)$ \\
incl. & $75^{\circ}$ & $35^{\circ}-45^{\circ}$ \\
$M_{1}\left[M_{\odot}\right]$ & $0.41^{a}(>0.70)^{b}$ & $0.55 \pm 0.15$ \\
$M_{2}^{d}\left[M_{\odot}\right]$ & 0.018 & 0.047 \\
$a\left[10^{10} \mathrm{~cm}\right]$ & 3.3 & 3.7 \\
$T_{\text {sh }}[$ years $]$ & $\sim 30$ & $\sim 8$ \\
\hline
\end{tabular}

${ }^{a} q$ derived from $K_{1} / V_{\mathrm{d}}$ (Smak 1993), given for consistency reasons.

${ }^{b}$ More reliable value obtained from $K_{2}$ (Steeghs et al. 2001).

c $q$ derived from $K_{1} / V_{\mathrm{d}}$.

$d$ From superhump excess.

$e$ Assumed.

(i.e. phase-binned) spectra, e.g. in Skidmore et al. (2000, their Fig. 1) and in our Fig. 3. The differences may not be explained as simple inclination effects Sect. 3.3: (i) the separation of the components in WX Cet is considerably less than in WZ Sge and (ii) the amplitude of S-Wave in WX Cet remains large compared to separation of disc components. For any reasonable white dwarf masses observation (i) and the lack of eclipses favours small inclination $i$. However, observation (ii) is insensitive to inclination and indicates deep penetration of the disk by the stream. Note that the estimate of $M_{1}$ rests mainly on the well-measured disc rotation $V_{\mathrm{d}}$ and on the inclination $i$. For a known distribution of $i$, e.g. for eclipsing CBs or for a sample free of inclination selection effects, measurements of $V_{\mathrm{d}}$ yield a reliable average mass for the primaries. Use of either $K_{1}$ or the superhump $q-\epsilon$ correlation for mass determination yields a secondary mass $M_{2}$ in WX Cet which is consistently large compared to that in WZ Sge. This demonstrates that WX Cet is not as advanced in its evolution as WZ Sge. Only the latter star has chance of being past the 80 -min minimum period stage.

Acknowledgements. First of all we would like to thank John Thorstensen for comments and access to his spectroscopic data.
We are grateful to Joe Smak for his calculations and for generous discussions. Comments on the evolution of the binary by Bohdan Paczynski and Lev Yungelson are mostly appreciated. This work was supported by KBN Grants 2P03D01818 and 5P03D00420.

\section{References}

Augusteijn, T., Heemskerk, M. H. M., Zwarthoed, G. A. A., \& van Paradijs, J. 1994, A\&AS, 107, 219

Bailey, J. 1979, MNRAS, 189, 41P

Baptista, R., Steiner, J. E., \& Cieslinski, D. 1994, ApJ, 433, 332

Cheng, F. H., Sion, E. M., Szkody, P., \& Huang, M. 1997, ApJ, 484, L149

Clemens, J. C., Reid, I. N., Gizis, J. E., \& O’Brien, M. S. 1998, ApJ, 496, 352

Downes, R. A. 1990, AJ, 99, 339

Downes, R. A., \& Margon, B. 1981, MNRAS, 197P, 35

Gilliland, R. L., Kemper, E., \& Suntzeff, N. 1986, ApJ, 301, 252

Howell, S. B., Szkody, P., \& Canizzo, J. K. 1995, ApJ, 439, 337

Krzeminski, W., \& Kraft, R. P. 1964, ApJ, 140, 921

Livio, M., \& Iben, I. 1993, PASP, 105, 1373

Lubow, S. H. 1992, ApJ, 401, 317

Mennickent, R. 1994, A\&A, 285, 979

O’Donoghue, D., Chen, A., Marang, F., et al. 1991, MNRAS, 250, 363

Osaki, Y. 1985, A\&A, 144, 369

Osaki, Y. 1989, PASJ, 41, 1005

Paczynski, B. 1977, ApJ, 216, 822

Paczynski, B., \& Schwarzenberg-Czerny, A. 1980, Acta Astron., 30, 127

Paczynski, B., \& Sienkiewicz, R. 1981, ApJ, 248, 27

Patterson, J. 1998, PASP, 110, 1132

Patterson, J. 2001, PASP, 113, 736

Rogoziecki, P., \& Schwarzenberg-Czerny, A. 2001, MNRAS, 323, 850

Skidmore, W., Mason, E., Howell, S. B., et al. 2000, MNRAS, 318, 429

Smak, J. 1993, Acta Astron., 43, 101

Smak, J. 2001a, Acta Astron., 51, 295

Smak, J. 2001b, Acta Astron., 51, 279

Steeghs, D., Marsh, T., Knigge, C., et al. 2001, ApJ, 562, L145

Strohmeier, W. 1964, IBVS 47

Thorstensen, J., Patterson, J., Shambrook, A., \& Thomas, G. 1996, PASP, 108, 73

Vogt, N. 1979, A\&A, 77, 7

Warner, B. 1995, Cataclysmic Variable Stars (Cambridge University Press)

Whitehurst, R. 1988, MNRAS, 233, 529 